\title{
A Research on Acoustic Characteristics of Gas Pressure Regulator with Silencer
}

\author{
Aleksandr A. Igolkin, Aleksandr N. Kovartsev and Dmitry M. Stadnik \\ Samara State Aerospace University, Moskovskoe shosse, 34, Samara, 443086, Russian Federation.
}

(Received 4 August 2015; accepted 30 June 2016)

Results of calculations and experimental studies of the acoustic characteristics of the gas pressure regulator with a silencer are reported here. A mathematical model and a method for obtaining a stable solution of the original system of equations was developed. The possibility of reducing the noise of the pressure regulator, when one orifice plate is installed, is presented here. We obtained a good convergence of theoretical and experimental data. The optimization of the flow area silencer consisting of several orifice plates was developed.

\section{NOMENCLATURE}

$\begin{array}{ll}D & \text { Coefficient of viscosity } \\ d & \text { diameter } \\ F & \text { force } \\ G & \text { mass flow } \\ J & \text { spring stiffness } \\ k & \text { heat capacity ratio } \\ l & \text { length } \\ M & \text { mass } \\ V & \text { volume } \\ R & \text { gas constant } \\ S & \text { area } \\ T & \text { stagnation temperature } \\ x & \text { valve lift } \\ Z & \text { resistance } \\ \mu & \text { flow coefficient } \\ v & \text { kinematical viscosity } \\ p & \text { pressure }\end{array}$

\section{INTRODUCTION}

There is a large number of papers dedicated to reducing noise and vibration of pneumatic and gas transportation systems. Among the manufacturers of regulating and isolation valves, it is possible to identify a number of companies that successfully implement noise reduction means (NPO "Aurora" NPF "CKBA“, Fisher, Emerson, Samson, Tartarini, Masoneilan, Valin and others).

Two methods of aerodynamic noise reduction in regulating and isolation valves are shown by Monsen $\mathrm{J} .:^{1}$ step throttling and flow division (splitting into smaller jets). Silencers, soundproofing cases, and pipes coated with sound absorbing materials are some of the noise reduction means. Shakhmatov et al. $^{2}$ used an integrated gas pressure regulator noise reduction system along with a silencer and a soundproofing case.

Physical processes of aerodynamic noise generated by fittings are described by different researhes ${ }^{3,4}$ in details. Karczub et al. ${ }^{5}$ described a method of reducing noise using orifice plate. The ten-step silencer is patented in the United States. ${ }^{6}$ Moreover, the choice of passage sections/number of stages is

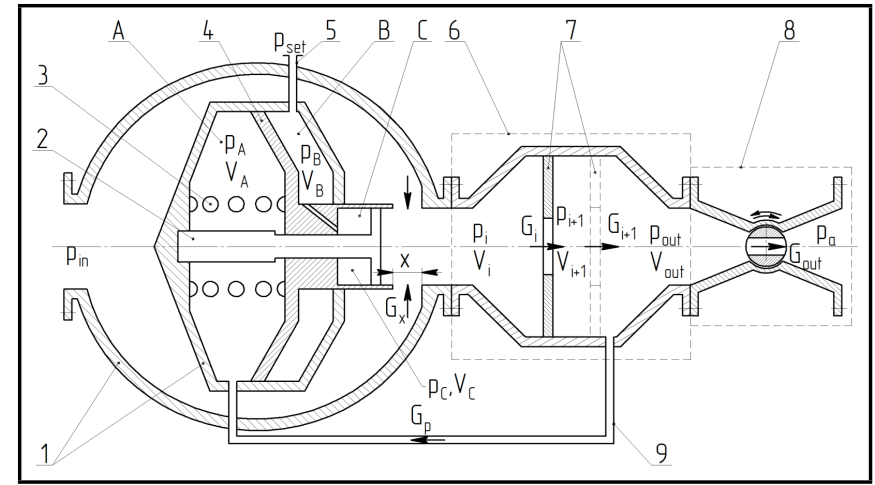

Figure 1. Schematic diagram of the gas pressure regulator with silencer: 1 regulator body; 2 - guide rod; 3 - spring; 4 - piston-valve; 5 - pressure adjusting supply line; 6 - silencer; 7 - a package of orifice plates; 8 - the valve; 9 feedback pipeline.

substantiated on the basis of initial pressure ratio. A qualitative description and visualization of the impact of the distances between jets on the overall noise level is provided in the Fisher company advertising catalogue. ${ }^{7}$ Among local scientists, we can distinguish following works. ${ }^{7-9}$ However, none of these papers discuss the choice of flow area of orifice plates and their impact on the operation of the pressure regulator.

\section{MATHEMATICAL MODEL}

Gas pressure regulators are widely used in gas distribution stations. However, due to the significant pressure drops during throttling and the appearance of high speeds, the work of these units is often accompanied by noise that exceeds the established health standards. There is a need to study the influence of the constructive solution on the acoustic characteristics of the regulator. One of the famous noise reduction methods is the installation of orifice plate at the outlet of the regulator. ${ }^{2,4,5}$ Schematic diagram of the system is shown in Fig. 1.

In the development of a mathematical model for the study of the acoustic characteristics of the system, "the regulator silencer", the following assumptions were made: a working fluid is an ideal gas; nonlinear friction forces are absent; input impedances of downstream systems are equal to zero $\left(p_{a}=\right.$ const).

Considering that the state of the gas in the cavities of the regulator and silencer changes according to adiabatic law, we 
can write:

$$
C_{i+1} \cdot \frac{d p_{i+1}}{d t}=G_{i}-G_{i+1}
$$

where $C_{i+1}=\frac{V_{i+1}}{k \cdot R \cdot T_{i+1}}$ is acoustic capacitance.

The equation of the gas motion in the pipeline 9 in lumped parameters with the account of hydraulic losses is as follows:

$$
\left(p_{\text {out }}-p_{A}-Z_{\text {pipe }} \cdot G_{\text {pipe }}\right) \cdot \frac{S_{\text {pipe }}}{l_{\text {pipe }}}=\frac{d G_{\text {pipe }}}{d t} ;
$$

where $Z_{p}=\frac{128 \cdot \nu \cdot l_{p}}{\pi \cdot d_{p}^{4}}-$ hydraulic resistance of pipeline 9 at laminar flow.

Gas flow through the i-th throttling cross section is described by Saint-Venant and Wenzel equation:

$G_{i}=\mu_{i} \cdot S_{i} \cdot p_{i} \cdot \sqrt{\frac{2}{R \cdot T_{i}} \cdot \frac{k}{k-1} \cdot\left[\left(\frac{p_{i+1}}{p_{i}}\right)^{\frac{2}{k}}-\left(\frac{p_{i+1}}{p_{i}}\right)^{\frac{k+1}{k}}\right]} ;$

when $\frac{p_{i+1}}{p_{i}}>\beta_{\text {critical }}$ subcritical flow regime,

$$
G_{i}=\mu_{i} \cdot \pi \cdot S_{\mathrm{i}} \cdot p_{i} \cdot \sqrt{\frac{k}{R \cdot T_{i}}} \cdot\left(\frac{2}{k+1}\right)^{\frac{k+1}{2 \cdot(k-1)}}
$$

when $\frac{p_{i+1}}{p_{i}} \leq \beta_{\text {critical }}$ supercritical flow regime.

The flow rate through the valve at the output from the system is determined in the same way.

The equilibrium equation of the actuating element of the pressure regulator can be written as follows:

$$
\begin{aligned}
M \cdot & \frac{d^{2} x}{d t^{2}}+D \cdot \frac{d x}{d t}+J \cdot x-p_{B} \cdot\left(\mathrm{S}_{\mathrm{p}}-\mathrm{S}_{\mathrm{f}}\right)- \\
& -p_{c} \cdot\left(\mathrm{S}_{\mathrm{f}}-\mathrm{S}_{\mathrm{rod}}\right)+\mathrm{F}_{0}+p_{A} \cdot\left(\mathrm{S}_{\mathrm{p}}-\mathrm{S}_{\mathrm{rod}}\right)=0 ;
\end{aligned}
$$

where $S_{p}, S_{f}, S_{\text {rod }}$ - area of the piston, flap, and rod; $F_{0}$ spring pretension force.

From Eq. (5), for the stationary case, when $\frac{d p_{i+1}}{d t}=0$, and the flow rate of gas through the valve can be determined using one of the formulas from Eq. (6) (see top of the next page).

Then for the stationary regime of flow, when $\frac{d p_{i+1}}{d t}=0$, a mathematical model of the pressure regulator containing $\mathrm{n}$ orifice plates, can be written by the following system of nonlinear equations:

$$
\left\{\begin{array}{l}
G_{x}-G_{1}=0 \\
G_{1}-G_{2}=0 \\
\cdots \\
G_{n}-G_{\text {out }}=0 .
\end{array}\right.
$$

The pressure in chambers between throttles of the system can be calculated from Eq. (3), (4), (6), and (7).

To calculate the acoustic power generated by a valve or an orifice plate, the Dr. Hans D. Baumann model was used, which is given in the monograph edited by L. Beranek: ${ }^{4}$

$$
W_{i}=\eta_{i} \cdot 7.7 \times 10^{-11} C_{\nu i} \cdot F_{L i} \cdot c_{0}^{3} \cdot p_{i} \cdot G_{f} ;
$$

where - speed of sound; - acoustic efficiency; - pressure recovery coefficient; - valve flow coefficient; Gf - specific gravity of the vapor or gas (relative to air $\mathrm{Gf}=1$ ). The acoustic efficiency for different flow regimes is defined in Eq. (9) (see top of the next page).
The valve flow coefficient can be determined by

$$
C_{\nu i}=\left\{\begin{array}{lr}
2.14 \times 10^{7} \cdot \frac{G_{i}}{\sqrt{\Delta p \cdot\left(p_{i}+p_{i+1}\right) \cdot G_{f}}} & \Delta p \leq \frac{1}{2} F_{L}^{2} \cdot p_{i}, \\
1.95 \times 10^{7} \cdot \frac{G_{i}}{F_{L i} \cdot p_{i} \cdot \sqrt{G_{f}}} & \Delta p>\frac{1}{2} F_{L}^{2} \cdot p_{i},
\end{array}\right.
$$

where $\Delta p=p_{i}-p_{i+1}$.

Similar expressions are obtained for each of the orifice plates.

Let $\bar{S}=\left(\bar{S}_{1}, \bar{S}_{2}, \ldots, \bar{S}_{n}\right)$ be a vector of relative flow areas of orifice plates. It is clear that the choice of the flow areas of orifice plates $S_{i}$ should be made depending on the minimum of the total noise $W_{\sum}$ of the regulator silencer system. The optimization problem is also solved, which can be formulated as a problem of the conventional global optimization:

$$
\left\{\begin{array}{l}
\left.\bar{S}_{\text {opt }}=\arg \min _{s} W_{\Sigma}(\bar{S})\right) \\
\bar{S}_{i} \in[0,1] ; \\
p_{i}>p_{i+1}, \quad i=\overline{1, n}
\end{array}\right.
$$

where $n$ - number of orifice plates of the silencer.

\section{METHOD OF OBTAINING A STABLE SO- LUTION OF THE SYSTEM OF EQUATIONS}

Despite the apparent simplicity of the model of a pressure regulator with a silencer, it contains a lot of technical difficulties that hinder the usage of standard numerical methods of MATLAB. ${ }^{11}$ In particular, in a strict representation of the mathematical model of a regulator with a silencer Eq. (7), we need to supplement the system of nonlinear Eq. (7) by the system of inequalities $p_{i}>p_{i+1}, \quad i=\overline{1, n}$ to ensure the correct usage of expressions (1) to ((6). Otherwise, the convergence conditions of known algorithms for solving system of nonlinear equations ${ }^{12}$ are violated. As a result, the algorithm "fsolve“" of MATLAB cannot find a solution or it finds a solution in the complex domain that does not have physical meaning.

On the other hand, in the numerical solutions of Eq. (7), a critical situation may arise when $p_{i}<0$.

The avoidance of these effects is possible by the introduction of nonlinear transformations of the independent variables.

For the pressure vector $\mathrm{p}$ we introduce new variables:

$$
\left\{\begin{array}{l}
x_{1}=p_{1} / p_{i n} \\
x_{i+1}=p_{i+1} / p_{i}, \quad \overline{i=1, n}
\end{array}\right.
$$

where $p_{n+1}=p_{\text {out }}$.

For new variables $x_{i} \in[0,1]$ is valid. The transformation of Eq. (12) provides an automatic fulfilment of the conditions: $p_{i}>p_{i+1}, i=\overline{1, n}$. Next transformation takes the range of independent variables of the system (7) to the whole space.

$$
z_{i}=-\ln \left(\left(1-x_{i}\right) / x_{i}\right), \quad i=\overline{1, n}
$$

In this case, $z_{i} \in(-\infty, \infty)$ greatly simplifies the task of solving a system of nonlinear Eq. (7); moreover, formulation of the problem of global optimization Eq. (11) is significantly simplified, because the part of the restriction is removed.

Backward transformation:

$$
\left\{\begin{array}{l}
x_{i}=\frac{1}{1+e^{-z_{i}}} \\
p_{1}=x_{1} \cdot p_{i n} \\
p_{i+1}=x_{i+1} \cdot p_{i}, \quad i=\overline{1, n} ;
\end{array}\right.
$$




$$
\begin{gathered}
G_{x}=\mu_{x} \cdot \pi \cdot d \cdot x \cdot p_{\text {in }} \cdot \sqrt{\frac{k}{R \cdot T_{\mathrm{in}}}} \cdot\left(\frac{2}{k+1}\right)^{\frac{k+1}{2 \cdot(k-1)}} \text { when } \frac{p_{1}}{p_{i n}} \leq \beta_{\text {critical }} ; \\
G_{x}=\mu_{x} \cdot \pi \cdot d \cdot x \cdot p_{i n} \cdot \sqrt{\frac{2}{R \cdot T_{\mathrm{in}}} \cdot \frac{k}{k-1} \cdot\left[\left(\frac{p_{1}}{p_{i n}}\right)^{\frac{2}{k}}-\left(\frac{p_{1}}{p_{i n}}\right)^{\frac{k+1}{k}}\right]} \text { when } \frac{p_{1}}{p_{i n}}>\beta_{\text {critical }} . \\
\left\{\begin{array}{c}
\eta_{I}=10^{-4} \cdot F_{L}^{2} \cdot\left(\frac{p_{i}-p_{i+1}}{p_{i} \cdot F_{L}^{2}-p_{i}+p_{i+1}}\right)^{2.6} \text { when } \frac{p_{i}}{p_{i+1}}<\left(\frac{p_{i}}{p_{i+1}}\right)_{\text {critical }} ; \\
\eta_{I I, I I I}=10^{-4} \cdot F_{L}^{2} \cdot\left(\frac{p_{i} / p_{i+1}}{p_{i} / p_{i+1 \text { critical }}}\right)^{3.7} \text { when }\left(\frac{p_{i}}{p_{i+1}}\right)_{\text {critical }} \leq \frac{p_{i}}{p_{i+1}}<3.2 \cdot \alpha_{p} ; \\
\eta_{I V, V}=1.32 \cdot 10^{-3} \cdot F_{L}^{2} \cdot\left(\frac{p_{i} / p_{i+1}}{\left(\frac{p_{i}}{p_{i+1}}\right)_{\text {break }}}\right)^{3.7} \text { when } 3.2 \cdot \alpha_{p} \leq \frac{p_{i}}{p_{i+1}}<22 \cdot \alpha_{p} ;
\end{array}\right.
\end{gathered}
$$

where $\left(\frac{p_{i}}{p_{i+1}}\right)_{b r e a k}$ is the critical pressure ratio, which depends on the design of the device and the working fluid; $\alpha$ - pressure recovery correction factor, which is based on the value of the pressure recovery coefficient. ${ }^{4}$

makes it possible to realize the transition from the "technological" independent variables to the original settings of the system $p_{i}$.

Finally, to improve the efficiency of the optimization algorithm, the constrained optimization problem (11) can be reduced to the problem of unconstrained optimization by the transformation process.

$$
y_{i}=-\ln \left((b-a) /\left(\bar{S}_{i}-a\right)-1\right), \quad i=\overline{1, n} ;
$$

where $a, b$ - are the boundaries of the domain of the optimized variables $\bar{S}_{i} \in[a, b]$. In our case, $a=0.05, b=0.95$. As a result, constrained optimization problem Eq. (11) is transformed into unconstrained optimization due to transformations (12), (13), (15).

$$
\left.y_{o p t}=\arg \min _{y} W_{\Sigma}(y)\right) .
$$

The proposed transformation of variables (12), (13), and (15) makes it possible to move from vectors of the optimized variables $\bar{S}_{i}$ of the task (11) and independent variables $p$ of the nonlinear equations system (7) to new variables belonging to the whole real axis, without disrupting the continuity of functions of equations system and criteria for evaluating the effectiveness of the designed system, since all transformations are continuous monotonic functions. This condition is significant because the Jacobian of a vector function of the system of equations is automatically calculated in the algorithm fsolve, i.e., first partial derivatives. The most important thing is that under arbitrary combinations of the vector of new variables $\mathrm{z}$ for the original pressure vector $\mathrm{p}$ constraint conditions are automatically satisfied (11).

Numerical experiments with model (7) for the new independent variable $\mathrm{z}$ confirmed the high efficiency of the proposed system of coordinate transformation. The algorithm demonstrated reliable performance for different combinations of input data.

Figure 2 shows the dependence of acoustic power for the system "pressure regulator +2 orifice plates", obtained using the developed method. Thus, the use of the proposed method allows to find dependences having a clear physical interpretation.

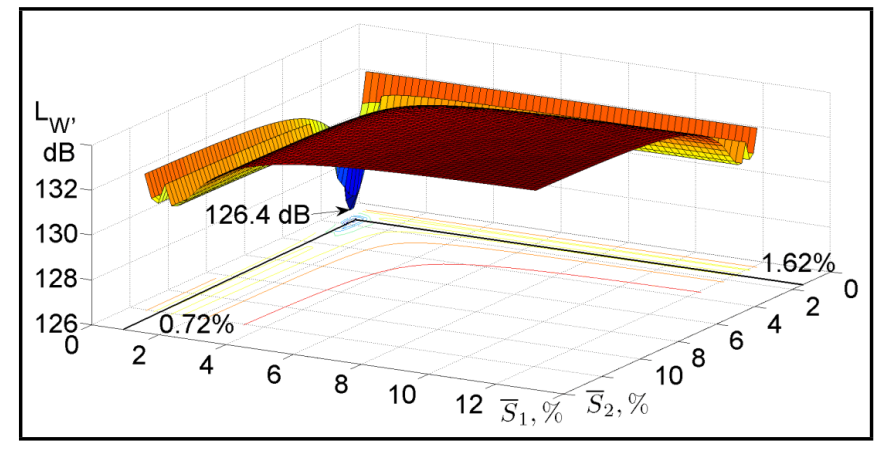

Figure 2. Dependence of acoustic power for the system "Regulator pressure + 2 orifice plates" obtained using the developed method.

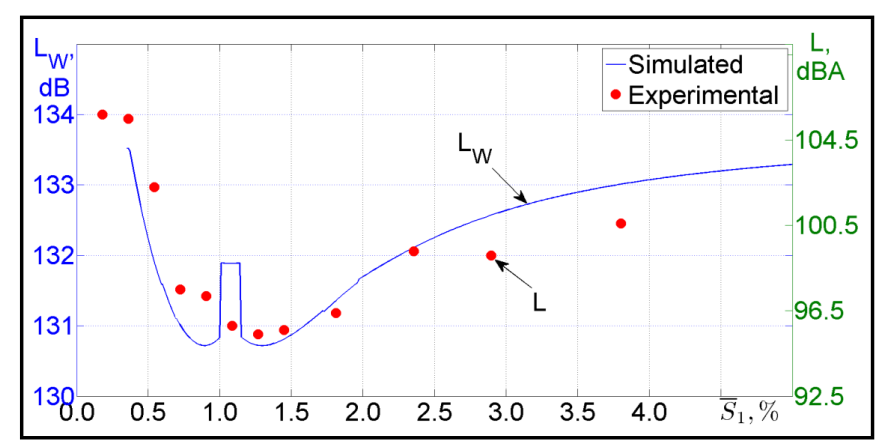

Figure 3. Dependence of sound power levels $L_{w}$ on the flow area of the orifice plate $\bar{S}_{1}=\frac{S_{1}}{S_{m}}$, where $S_{m}$ silencers area.

\section{EXAMPLE OF CALCULATION AND COM- PARISON WITH EXPERIMENTAL DATA}

Consider the solution of the optimization problem as an example of the "pressure regulator with a silencer consisting of an orifice plate“ (Fig. 1).

The results obtained for these parameters are shown in Fig. 3: $p_{\text {out }}=40 \mathrm{~Pa} ; p_{f}=4.0 \mathrm{TI} ; d_{\text {out }}=18 \mathrm{~mm}$.

Minimum of the acoustic power generated by two sources (a regulator and an orifice plate) is achieved at a relative flow area of orifice plate $1-1.5 \%$, which agrees well with the measured sound pressure level at $1 \mathrm{~m}$ from the pipeline.

The method developed makes it possible to find the optimal flow areas of orifice plates practically for any number of 


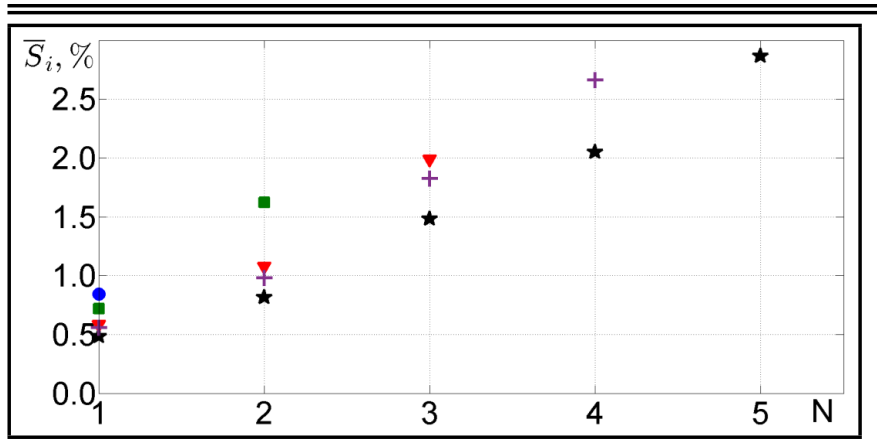

Figure 4. Dependence of flow areas of orifice plates $S_{i}$ on the number $N$ of orifice plates.

orifice plates. In particular, the results of the computational experiment of a silencer, containing 1 to 5 orifice plates is shown in Fig. 4.

The optimum flow area of the orifice plate is determined by the minimum value of acoustic power level. At this same time, the acoustic power of the system is the amount of power generated by the valve and orifice plates. For example, minimum of acoustic power of the system with a silencer, consisting of one orifice plate (indicated round shape blue marker), is observed in its flow area $\bar{S}_{1}=0.84 \%$. This value is determined by the intersection point of two curves: sound power level generated by the valve and the orifice plate (see. Fig. 3). This principle can be applied to a system with a silencer consisting of several orifice plates.

The figure shows that if the silencer consists of $n$ orifice plates, then the flow area of the following orifice plate is bigger than the previous. For example, for the silencer which consists three orifice plates (labeled markers triangular shape red), flow areas are: $0.581 \%, 1.075 \%$, and $1.985 \%$. The flow area of follow orifice plate increases $\bar{S}_{i+1}>\bar{S}_{i}$ due to the pressure drop when extra orifice plate is installed in the silencer $\left(p_{i}>\right.$ $\left.p_{i+1}\right)$.

Extra orifice plate in a system with silencer leads to a pressure drop at the output $p_{n+1}$ and in the chamber A; therefore, the flow area of the valve will increase (see. Fig. 1). So the pressure $p_{1}$ between the valve and orifice plate will increase. Considering that the air consumption through the system is constant, silencer flow area is reduced (see. Fig. 4).

\section{CONCLUSIONS}

1. A mathematical model of the gas pressure regulator with a silencer was developed. The impact of orifices on the acoustic characteristics of the system was analyzed based on this model.

2. A method for obtaining a stable solution of nonlinear equations was proposed. It was used to calculate the parameters of the pneumatic system and the optimal values of flow area of orifice plates in terms of minimizing the generated acoustic power.

3. In examples of the silencer with one and two orifice plates, graphs of the acoustic power vs. their areas were drawn, which demonstrated the existence of "optimums", characterized by a minimum of acoustic power radiated by the system.
4. It was determined that the optimal values of flow area of orifice plates are in the range of one to five, and analysis of these orifice plates revealed a tendency of area increment for each subsequent orifice plate.

5. The experimental results agree well with the calculated data.

\section{ACKNOWLEDGEMENTS}

This work was supported by the Ministry of Education and Science of the Russian Federation.

\section{REFERENCES}

1 Monsen J. Valve Noise Reduction Strategie, http: //www.valin.com/resources/whitepapers/ valve-aerodynamic-noise-reductionstrategies, (Accessed Juily 25, 2011).

2 Igolkin .., Kruchkov A.N., Koh A.I., Safin A.I. and Shakhmatov E.V. Pressure reducing valve noise reduction A. Proceedings of the Nineteen International Congress on Sound and Vibration (ICSV 19), 3, 2458-2464 (2012).

3 Flod D. Jury. Fundamentals of Aerodynamic Noise in Control Valves. Technical monograph 43, (1999).

4 Istvan, L, Noise and Vibration Control Engineering: Principles and applications, New Jersey, (2006).

5 Denis G. Karczub, Fred W. Catron and Allen C. Blowdown valve noise and interactions with down stream orifice plates. Proceedings of 2003 ASME International Mechanical Engineering Congress, 43-49, (2003). https://dx.doi.org/10.1115/IMECE2003-55111

6 A Trevor Smith, Fluid-flow noise reduction systems, US 4113050, British Gas Corporation, (1978).

7 The Silent Treatment Fisher. Solutions to Noise Problems, http: //www. spartancontrols.com/ /media/ resources/fisher/b/186_fisher_brochure. pdf, (Accessed Febrary, 2012).

8 Makaryants, G. M., Sverbilov, V. Y., Prokofiev, A. B., Makaryants, M. V., and Shakhmatov, E. V. The tonal noise reduction of the proportional pilot-operated pneumatic valve. Proceedings of the Nineteen International Congress on Sound and Vibration (ICSV 19), 1, 689-696 (2012).

9 Arzumanov E.S. Hydraulic regulators of automatic control systems, Moscow, (1985).

10 Tolokonnikov I.S., Karmanova V.V., Oleynikov A.Yu. et al. Snizheniye urovney shuma i vibratsii na gazoraspredelitel'nykh punktakh energeticheskikh ob"yektov, Sbornik dokladov III Vserossiyskoy nauchno-prakticheskoy konferentsii s mezhdunarodnym uchastiyem, 245-260, (2011).

11 D'yakonov V.P. MATLAB 6/6.1/6.5 + Simulink application in matematiks and simulation, (2003).

12 Kovartsev A.N. Mathematics of computations. Samara, (2011). 